\title{
Propuesta de un método para el uso de juegos serios en la conservación del patrimonio natural y cultural
}

\author{
Iroel Heredia \\ Programa de Maestría y Doctorado en Arquitectura \\ Universidad Nacional Autónoma de México \\ Ciudad de México, México \\ iroel.unam@gmail.com
}

\begin{abstract}
En este trabajo se estudia la problemática de la perdida de patrimonio natural, cultural y arquitectónico de los pueblos originarios y se plantea un método para la preservación de este patrimonio mediante el uso de los juegos serios y las TIC's, en el marco del codiseño y el respeto a los valores socioculturales de las comunidades.
\end{abstract}

\section{Keywords-Juegos Serios, Conservación Patrimonio,} Realidad Mixta, Realidad Virtual

\section{INTRODUCCIÓN}

La importancia del uso de las tecnologías actuales para la preservación del patrimonio ha sido ampliamente discutida en múltiples foros, sin embargo, es un hecho que la memoria material, cultural, arquitectónica e incluso natural se sigue perdiendo, especialmente en los pueblos indígenas, y es por esto por lo que se propone un método que, mediante el uso de los Juegos Serios, ayude a recuperar el valor que tiene este patrimonio para fomentar su cuidado y conservación.

\section{MotivacióN}

El patrimonio natural y cultural de las comunidades autóctonas, especialmente de las pequeñas comunidades, se pierde cada año por múltiples factores, uno de ellos es la alienación y desconocimiento de su valor por las nuevas generaciones, las cuales cambian los usos y costumbres de sus pueblos, por los valores de la sociedad centralizada, intentando integrarse a la actualmente llamada sociedad de la información. Este desconocimiento como menciona el antropólogo Arjun Appadurai [1] reduce su valor percibido y por lo tanto la intención de su cuidado

Por otro lado en la sociedad moderna el conocimiento es transmitido muchas veces por medios electrónicos y los jóvenes que son parte de las generaciones denominadas "Nativos digitales" (Millenial, y Alpha) [2] tienden a pensar que lo que se entrega mediante medios electrónicos es avalado, y por lo tanto superior, a otras formas de conocimiento. Aunque esta forma de pensar en las zonas rurales aún no está completamente permeada, es parte del aspiracional de la población e impulsada por actores sociales como las compañías y el gobierno central.

\section{TRABAJO RELACIONADO}

Esta investigación se enmarca en la colaboración que tiene el Laboratorio de Conservación del Patrimonio Natural y Cultural de la Universidad Nacional Autónoma de México y el municipio de Santiago de Anaya en la búsqueda de conservar la cultura, arquitectura y relación con la región del pueblo Hñähñu. Hasta el día de hoy esta colaboración ha tenido como resultado dos trabajos de investigación concluidos, el primero de ellos es la Caracterización de las capillas de Santiago de Anaya, Hidalgo [3] y el segundo el Diseño de corredores eco-culturales en la misma región [4].
Estos trabajos han generado propuestas para el reconocimiento arquitectónico y cultural de la zona. Por otro lado, existen múltiples investigaciones donde la Realidad Mixta es utilizada para la preservación del patrimonio, entre ellos podemos encontrar el trabajo de Anderson llamado Serius Games in Cultural Heritage [5] o el de Mortara: Learning cultural heritage by serius games [6]en los cuales se describe el potencial del uso de los Juegos Serios como herramientas para la protección, documentación y conocimiento del patrimonio.

\section{PROpuesta de SOlución}

a) Se propone la creación de un método de diseño de productos digitales (realidad mixta) mediante Juegos Serios que permita la valorización del patrimonio natural y cultural de las comunidades autóctonas reflejando los valores propios de estas. Para ello se retoman los métodos de diseño participativo y codiseño pensado especialmente para mantener los valores socioculturales de los pueblos autóctonos, en estos métodos no solo se recopila información de los actores involucrados, sino que se les hace participes del proceso de creación, buscando garantizar con esto la adecuada interpretación de las necesidades, deseos y visión desde la perspectiva de la comunidad. La metodología además propone utilizar múltiples iteraciones cortas que repercutan en un producto digital eficaz orientado siempre hacia ser un juego serio.

b) El método propuesto consta de 6 fases, las cuales además de cubrir aspectos técnicos o de diseño, incluyen también factores humanos y antropológicos, requiriendo un grupo interdisciplinar para su ejecución. Este método se encuentra dividido en 3 etapas: conocimiento, desarrollo y ejecución.

c) La primera etapa se divide en 2 pasos, Acercamiento a la comunidad y Diálogo, esto para permitir comprender los valores propios de la población y las intenciones, así como formar vínculos duraderos que faciliten la creación del producto final, es decir del juego.

d) La etapa de Desarrollo, incluye el Proceso de codiseño y Prototipado, en iteraciones cortas como las propuestas por metodologías como Sprints, Agile, Lead, Design Thinking[7][8] que permiten obtener retroalimentaciones por parte de usuarios y hacer correcciones dentro del equipo de trabajo, esta etapa es la más exhaustiva y requiere de un mayor número de individuos ya que el diseñador del juego no deberá ser el único en tomar decisiones si no que requiere dialogar horizontalmente con especialistas de diversas áreas como son el diseño, la conservación del patrimonio, autoridades locales e incluso autoridades comunales, quienes podrán ayudar en el desarrollo del juego, así mismo se requiere desde esta etapa la participación de 
diversas instituciones para apoyar la creación del juego. En esta etapa se desarrollan evaluaciones de usabilidad, transmisión de conocimiento, interacción humano-maquina, y valores estético-productivos entre otros.

Finalmente, la etapa de Ejecución donde se realiza la Publicación del juego, y además un Seguimiento para verificar que los objetivos de su creación se cumplan, utilizando para ello indicadores cualitativos y cuantitativos desarrollados por el equipo multidisciplinar en conjunto con la comunidad, en caso de que no se cumplan dichos indicadores es posible regresar a la etapa anterior en un nuevo ciclo de mejora.

\section{Resultados Preliminares}

Hasta la fecha se ha trabajado en la etapa de conocimiento, tanto con la comunidad como a nivel teórico, y siguiendo el método antes descrito se han generado modelos tridimensionales del entorno y el espacio arquitectónico, así como la creación de las variedades vegetales correspondientes, generando un banco de información que será vital para la comprobación del modelo. Próximamente se continuará con la validación del método con el desarrollo de un juego con fines de preservación de la cultura Hñähñu.

\section{CONCLUSIÓN.}

La inclusión de una etapa de reconocimiento de las diversas culturas y el trabajo horizontal con estas en búsqueda de la creación de juegos serios evita desviaciones de interpretación que podrían impedir el objetivo de preservación del patrimonio natural y cultural perseguido en su creación, para esto se requiere un trabajo cercano con las comunidades que potencialice el uso de estos juegos y las tecnologías asociadas en la actualidad.

\section{REFERENCIAS}

[1] E. A. Appadurai, The Social Life of Things: Commodities in Cultural Perspective, Cambridge: Cambridge University Press, 1986.

[2] L.Gibson et.al. «Millennials and Technology:Addressing the Communication Gap in Education and Practice.» Organization Development Journal, 2014: 63-75

[3] D. Guzman, Caracterización de las capillas de Santiago de Anaya, Hidalgo, Universidad Nacional Autónoma de México: Tesis de Licenciatura en Arquitectura, 2018.

[4] R. Cuevas, Diseño de corredores eco-culturales en Santiago de Anaya, Hidalgo, Universidad Nacional Autónoma de México: Licenciatura en Arquitectura del Paisaje, 2019.

[5] E.F. Anderson, «Serious Games in Cultural Heritage,» de The 10th International Symposium on Virtual Reality, Archaeology and Cultural Heritage VAST - State of the Art Reports, Goslar, 2009.

[6] M. Mortara, «Learning cultural heritage by serious games,» de Journal of Cultural Heritage, 2014.

[7] J. Gothelf . «Lean vs Agile vs Design Thinking: Lo que realmente necesitas conocer para construir productos digitales con equipos de alto rendimiento», CreateSpace Independent Publishing Platform, 2017

[8] E.Lupton, «Design is Storytelling»,Cooper Hewitt, NY. 2017 